RUHUNAJOURNAL OF SCIENCE

Vol 10(1): 51-64, June 2019

eISSN: $2536-8400$

Faculty of Science

DOI: http://doi.org/10.4038/rjs.v10i1.50

University of Ruhuna

\title{
Development of a novel and benign emulsion paint binder from epoxidized soybean oil and dimethylol urea blend
}

\author{
Ayodele Akinterinwa*, Sunday Ogwuche, S.A. Osemeahon and \\ Abdulraheem O. Anumah \\ Department of Chemistry, Modibbo Adama University of Technology, Yola, Nigeria \\ *Correspondence: ayoterinwa@yahoo.com; (iD https://orcid.org/0000-0001-5236-9238 \\ Received: $11^{\text {th }}$ October 2018, Revised: 30 ${ }^{\text {th }}$ April 2019, Accepted: $18^{\text {th }}$ May 2019.
}

\begin{abstract}
To develop a novel emulsion paint binder, dimethylol urea (DMU) resin synthesized from low formaldehyde to urea stoichiometric ratio (2:1) was blended with epoxidized soybean oil (ESBO) with 2.56\% oxirane oxygen and 67\% iodine value conversion. ESBO/DMU blends were prepared by varying the amount of ESBO from 10-70\%. The blends were analyzed for formaldehyde emission, viscosity, refractive index, turbidity, density, gelation time and solubility in water, while the blends' films were analyzed for moisture uptake and melting point. Homogenous blends were obtained up to 50\% ESBO/DMU blend, while FTIR analyses show reactive ESBO/DMU blend at optimum blending. Formaldehyde emission and moisture uptake were reduced in the blends' film, while ESBO also act as plasticizer by introducing softness in the blends' film. Comparison of the properties of $40 \%$ ESBO/DMU optimum blend with other reported binders shows its good potentials and its capability to compete in the coating industry.
\end{abstract}

Keywords: binder, dimethylol urea, epoxidation, emulsion paint, Soybean oil.

\section{Introduction}

Health and environmental impacts of painting and coating cannot be overemphasized; as we must stay "beautiful internally" (healthy), to enjoy and appreciate the external beautification. When emulsion or oil paints dries on a substrate, water or organic thinner vapor is released respectively. Organic thinners also referred to as volatile organic compounds are hazardous, this is why the production of emulsion/water based paints is progressively being researched to obtain products with competitive properties compared to oil based paint so as to replace them and avoid or limit the hazardous release of volatile organic compounds to safe and acceptable levels in the environment. Properties of urea formaldehyde (UF) resin such as solubility in water, fast curing, formation of hard, colorless, glossy, electrical-resistant and water 
insoluble film selects it as a potential emulsion paint binder. However, intrinsic properties of the film such as brittleness, poor moisture resistance which will lead to short term failure, and a hazardous emission of formaldehyde are limiting factors in its application in large surface coating (Edoga 2006, Osemeahon et al. 2015). A trend of research is focused on various modifications of UF resin to ameliorate its impeding properties as efficient binder in the formulation of emulsion paint. Among these modifications are some reported modulations in the resin synthesis (Barminas and Osemeahon 2007, Suurpere et al. 2006, Osemeahon et al. 2015). The modulation to synthesize DMU from formaldehyde and urea using reaction stoichiometry ratio 2:1 respectively, was aimed at the reduction of formaldehyde emission from the resin (Osemeahon et al. 2015). Other modifications include copolymerization and blending with other resins (Edoga 2006; Osemeahon and Barminas 2007, Fink 2013, Osemeahon et al. 2015, Idowu-Oyewole et al. 2016).

Blending is a common procedure that leads to the formation of new substances with outstanding physical, chemical and mechanical properties, with novel applications (Pizzi et al. 2002). UF resins have been blended with various substances and the resultant products have shown various improvements in the intrinsic properties limiting the application of the pure resin. However, reports on DMU copolymer blends are scarce. Osemeahon et al. (2015) blended DMU with polystyrene; this increased moisture resistance, impact softness/flexibility but could not appreciably reduce formaldehyde emission from the film at optimum blend, hence retaining some challenges.

Soybean oil (SBO) is available and cheap. It is polyunsaturated with relatively high double bond, and these points of unsaturation present site for such reaction as epoxidation (Saithai et al. 2013). Epoxidation reaction introduce reactive oxirane ring in the structure of oil (Figure 1), thereby making the oil more interactive (Saithai et al. 2013, Cheng et al. 2015, Pantone et al. 2017). Epoxidized oils serve as plasticizer/softener in rigid/hard polymers. It has been involved in coatings formulation, one of these is acrylated ESBO involved in the production of an emulsion paint binder (Habib and Bajpai 2011).

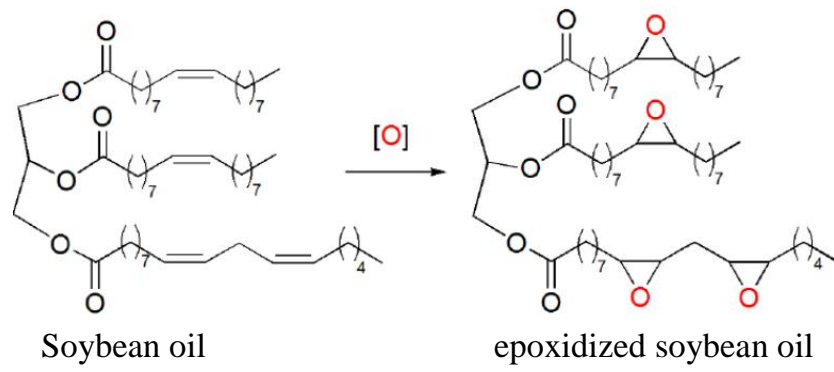

Fig. 1. Typical epoxidation reaction. (adopted from Pantone et al. 2017) 
In this study, ESBO was blended with DMU. A reactive homogenous blend expected from the two substances is anticipated to present a new product (ESBO/DMU) with tailored properties acceptable in coating application. ESBO/DMU is expected with potentials that will present a competitive alternative among the emulsion paint binder being considered in the coating industry.

\section{Material and Methods}

\subsection{Materials}

Chemicals used include Urea, formaldehyde, sodium dihydrogen phosphate, water, sulphuric acid, sodium hydroxide pellets, soybean oil, hydrogen peroxide (30 wt\%), glacial formic acid, $\mathrm{Na}_{2} \mathrm{~S}_{2} \mathrm{O}_{3}, \mathrm{NaOH}$ solution, lithium aluminate, dams reagent, starch indicator and phenolphthalein, pyridine, bromine, potassium iodide, sodium thiosulphate, chloroform, ethylether, hydrochloric acid and ethanol. All chemicals are analytical grade supplied by the British Drug House. Refined soybean oil by Agarwal Industries LTD, India, was purchased from the Market.

\subsection{Epoxidation of Soybean oil}

Method by Saithai et al. (2013) was adopted with slight modifications as follows. Soybean oil (100 g) was poured in to a $500 \mathrm{~mL}$ three neck flask equipped with reflux condenser assembled in a water bath with stirrer. Formic acid (13.97 g) was added and the mixture was stirred and heated up to $45^{\circ} \mathrm{C}$. Sulphuric acid $(0.5 \mathrm{~mL})$ was included in the mixture, the temperature was raised up to $60^{\circ} \mathrm{C}$, and hydrogen peroxide (116.98 g) was finally added drop wisely. Molar ratio of the soybean oil (double bonds in oil) to formic acid to hydrogen peroxide was determined to be 1:2.64:8.9. The reaction was allowed to run for 6 hours, cooled and the oil phase was decanted. The epoxidized oil was washed with warm distilled water and dried at $50^{\circ} \mathrm{C}$ in an oven for about 5 hours.

\subsection{Determination of Iodine value and conversion}

Iodine value of soybean oil before $\left(\mathrm{IV}_{0}\right)$ and after $\left(\mathrm{IV}_{\mathrm{t}}\right)$ epoxidation was determined according to Wijs method (AOAC 2000). Iodine value conversion (\% X) which also measures the relative percentage loss in un-saturation was calculated using Equation 1.

$$
\% X=\frac{I V_{0}-I V_{t}}{I V_{0}} \times 100
$$




\subsection{Determination of oxirane oxygen content}

This was carried out using the method by Siggia (1963). ESBO (0.20g) was quantitatively transferred into a conical flask. A hydro-chlorinated reagent was prepared from $0.2 \mathrm{M} \mathrm{HCl}$ and ethyl ether $(1: 1 \mathrm{v} / \mathrm{v})$, and $10 \mathrm{ml}$ was added into the conical flask and the mixture was allowed to stand for 3 hours. Blank solution was also prepared and the solutions were titrated with $0.1 \mathrm{M}$ sodium hydroxide solution using phenolphthalein as an indicator. Percentage oxirane content which also measures the stable epoxy groups on the epoxidized oil was calculated according to Equation 2.

$$
\text { Oxirane oxygen }=\frac{(B-S) \times M \times 16}{W \times 1000} \times 100
$$

Where, $\mathrm{S}=$ Volume of $\mathrm{NaOH}$ used for sample $(\mathrm{mL}), \mathrm{B}=$ Volume of $\mathrm{NaOH}$ used for blank (mL), M= Molarity of the $\mathrm{NaOH}, \mathrm{W}=$ Weight of sample (epoxidized oil) used (g) and $16 \mathrm{~g} / \mathrm{mol}=$ atomic mass of oxygen.

\subsection{Synthesis of DMU, ESBO/DMU blends and determination of physical properties}

Dimethylol urea (DMU) was synthesized according to the one step process (OSP) by Barminas and Osemeahon (2007), as modified in our previous work (Osemeahon et al. 2015). ESBO/DMU blends were prepared by a starring a mixture of 10, 20, 30, 40, 50, 60 and $70 \%$ ESBO in DMU resin, at room temperature $\left(30^{\circ} \mathrm{C}\right)$ till a homogenous mass was obtained.

Relative viscosity was determined at room temperature $\left(30^{\circ} \mathrm{C}\right)$ using a graduated glass macro-syringe standardized with $20 \%(\mathrm{w} / \mathrm{v})$ sucrose solution (viscosity $=2.0 \times 10^{-3} \mathrm{Nsm}^{-2}$ at $30^{\circ} \mathrm{C}$ ), and the gel time was recorded as the time at which a constant viscosity profile was obtained. Formaldehyde emission was determined using the standard 2 hour desiccators test. Moisture uptake form the atmosphere by the films was gravimetrically determined (Barminas and Osemeahon 2007). Density, turbidity (Hanna microprocessor turbidity meter, Model H193703), melting points (Galenkamp melting point apparatus Model MFB600-010F) and refractive index (Abbe refractometer) were carried out according to standard methods (AOAC 2000). In all analysis, averages of triplicate determinations were recorded.

\subsection{FTIR analysis}

Dimethylol urea (DMU) and ESBO/DMU blend was analyzed using infra-red spectrophotometer (Buck Scientific Inc, CT, USA Model M500) between 500 and $4000 \mathrm{~cm}^{-1}$. 


\section{Results and Discussion}

Iodine value of SBO $\left(\mathrm{IV}_{0}\right)$ was determined to be $84.8 \mathrm{~g}_{\mathrm{g}} \mathrm{I}_{2} / 100 \mathrm{~g}$ oil. After epoxidation $\left(\mathrm{IV}_{\mathrm{t}}\right)$ the value dropped to $32.36 \mathrm{~g}$ of $\mathrm{I}_{2} / 100 \mathrm{~g}$ oil and the iodine value conversion was calculated as $61.84 \%$. This indicated that un-saturation (double bonds) has been reduced in the oil. Percentage oxirane oxygen of the ESBO was obtained as $2.56 \%$, indicating that some point of un-saturation in the oil has been substituted by the oxirane oxygen.

\subsection{FTIR Spectra}

Figure 2 compares the spectra of ESBO/DMU blend with DMU. On DMU, peaks between $3700-3200 \mathrm{~cm}^{-1}$ attributes to absorption by $\mathrm{O}-\mathrm{H}$ and $\mathrm{N}-\mathrm{H}$, peaks within $2000-1000 \mathrm{~cm}^{-1}$ attributes to carbonyl $(\mathrm{C}=\mathrm{O})$ and $\mathrm{C}-\mathrm{O}-\mathrm{C}$ at the methylene ether linkages.

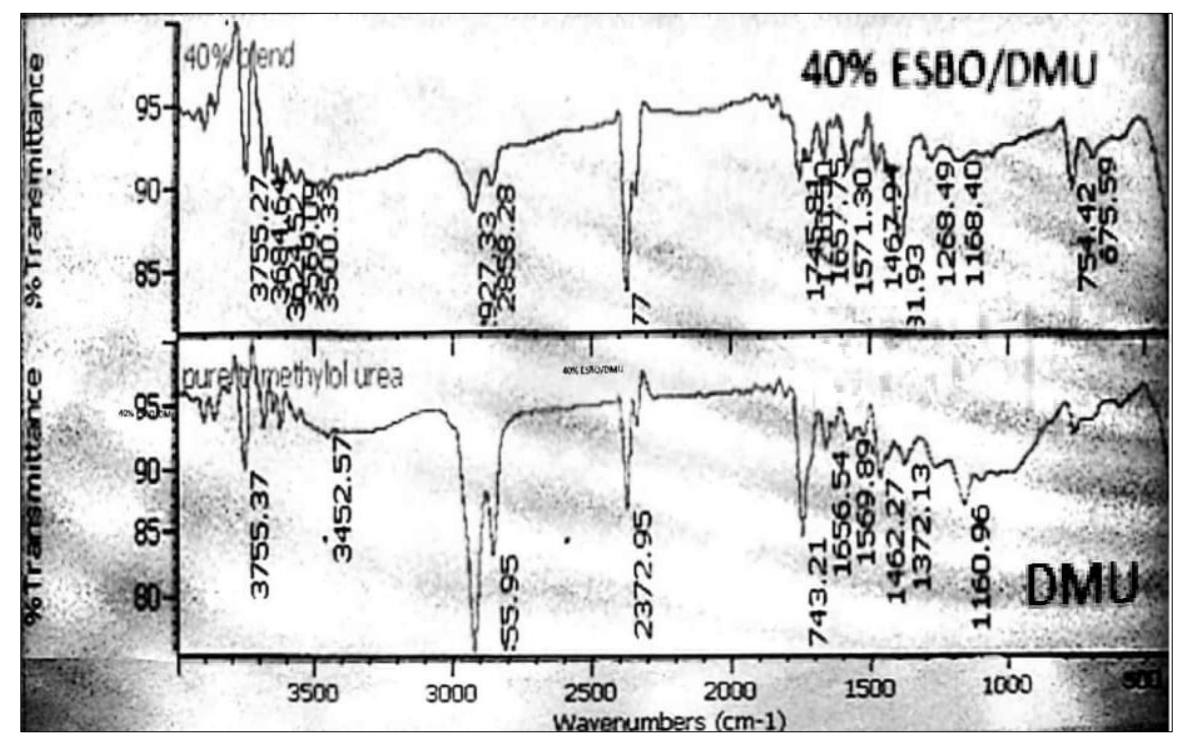

Fig 2. FTIR spectra of DMU and 40\% ESBO/DMU blend

The sharp peaks at between $3000-2500 \mathrm{~cm}^{-1}$ is due to the combined group ($\mathrm{NH}-\mathrm{CH}_{2}-\mathrm{OH}$ ) on the DMU resin (Figure 3). The blend's spectra show a significant change in intensity, and this may be attributed to interaction between ESBO and DMU. The reduction observed for the $-\mathrm{NH}-\mathrm{CH}_{2}-\mathrm{OH}$ peak indicates the involvement of this group in the interaction with ESBO as shown in Figure 3. There is no oxirane characteristic peak (at $820 \mathrm{~cm}^{-1}$ ) on the blend 
spectra; showing that the ESBO oxirane group could be involved in the interaction with DMU resin.

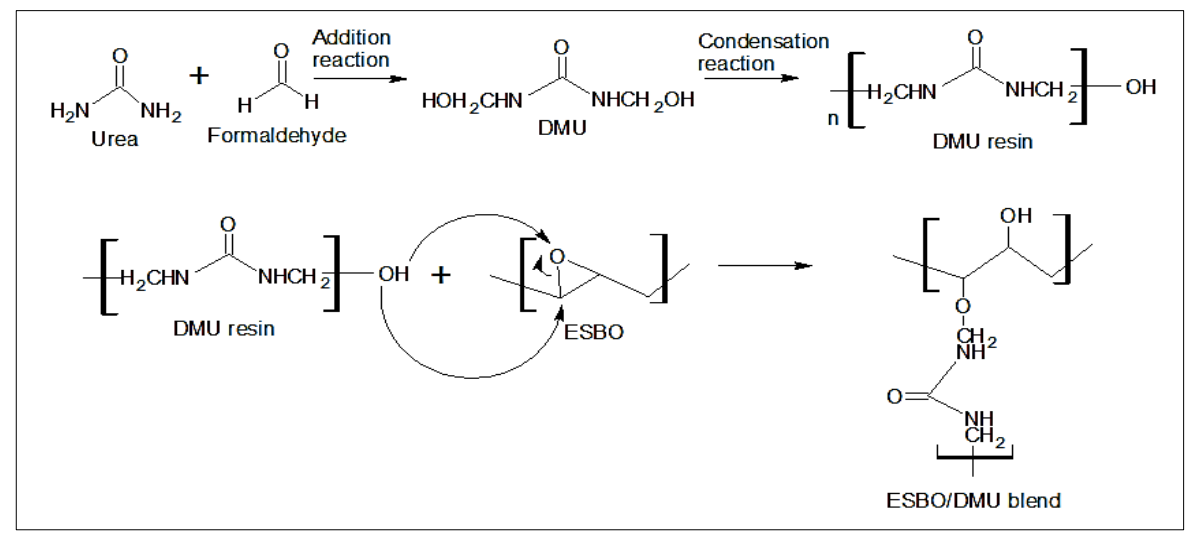

Fig. 3. Synthesis of DMU resin and a proposed interaction between ESBO and DMU in the blend.

\subsection{Effect of ESBO concentration on the physicochemical properties of ESBO/DMU blend}

Binder viscosity is a parameter that must be optimized for an optimum paint production and application processes, so as to correct paint leveling, sagging, flow and adhesion (Suurpere et al. 2006, Idowu-Oyewole et al. 2016). Figure 4 presents the effects of ESBO on the viscosity of the ESBO/DMU blend.

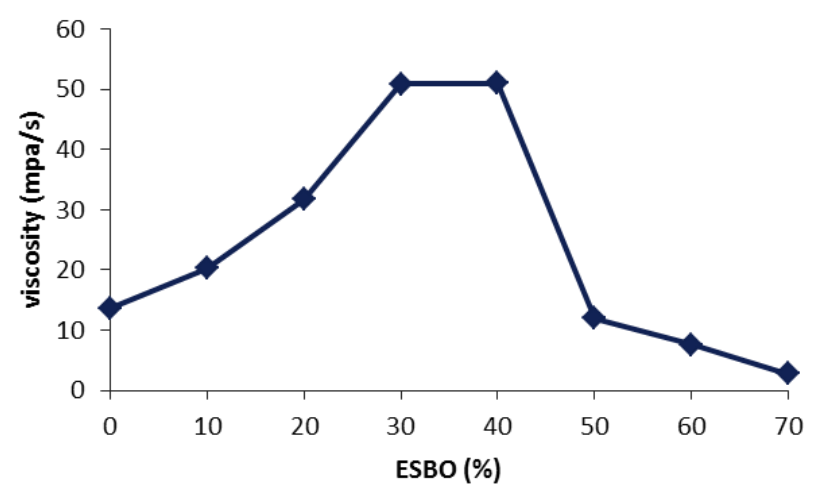

Fig. 4. Effect of ESBO content on the viscosity of ESBO/DMU blends 
The blend's viscosity increased with the inclusion and increase in the amount of ESBO up to 30\%, this peak viscosity was maintained till $40 \%$ after which a sharp drop in viscosity was observed. The initial effect can be attributed to homogeneity/interactions between ESBO and DMU leading to increase in the average molecular weight and dispersity index of the blend (Vilas et al. 2001, Oladipo et al. 2013). This can be said to reach optimum at the equilibrium concentration of ESBO, above which bulk of the blend is heterogeneous.

Binder density influence many important properties of the paint formulated with it (Idowu- Oyewole et al. 2016). The change in density of ESBO/DMU blends with variation in the ESBO content is presented in Figure 5. Increase in ESBO gradually decreases the density of the blends. This can be attributed to increase in the specific and free volume in the blended polymer consequent of changes in the morphology of DMU and the blends as ESBO increases in the blends (Osemeahon et al. 2015). Osemeahon et al. (2015) reported similar observation for a blend of DMU/polystyrene resin.

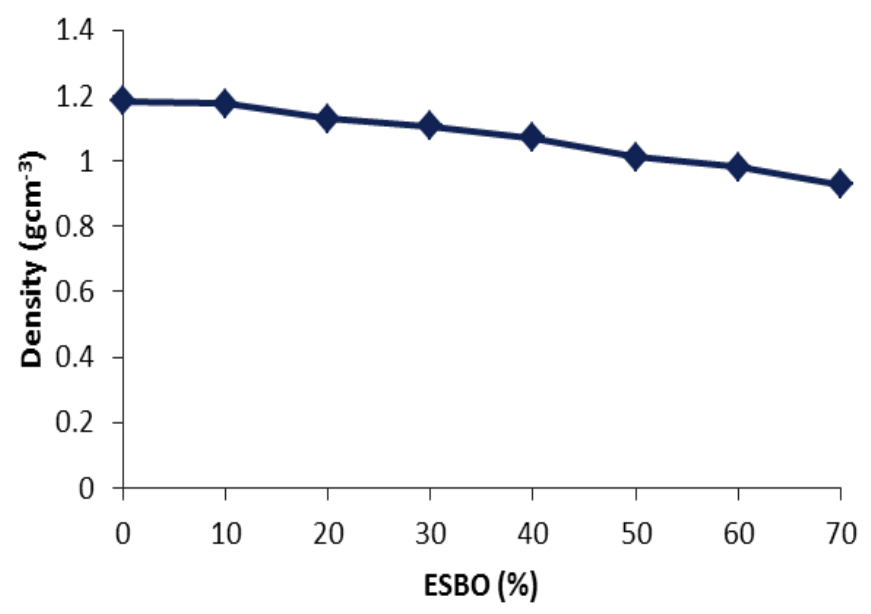

Fig 5. Effect of ESBO content on the density of ESBO/DMU blends

Optical properties of binder may influence the paint's aesthetic properties (Oladipo et al. 2013). The effect ESBO variations on the optical property of the blends were presented for refractive index and turbidity in Figures 6 and 7 respectively. Figure 6 showed a sharp rise in the refractive index of the blend at 10\% ESBO (inclusion of ESBO), followed by a gradual increase till $50 \%$. This may be due to increasing homogeneity/interaction up till 50\% ESBO leading to increase in light scattering boundaries and discontinuities in the blends (Liem et al. 2002). 


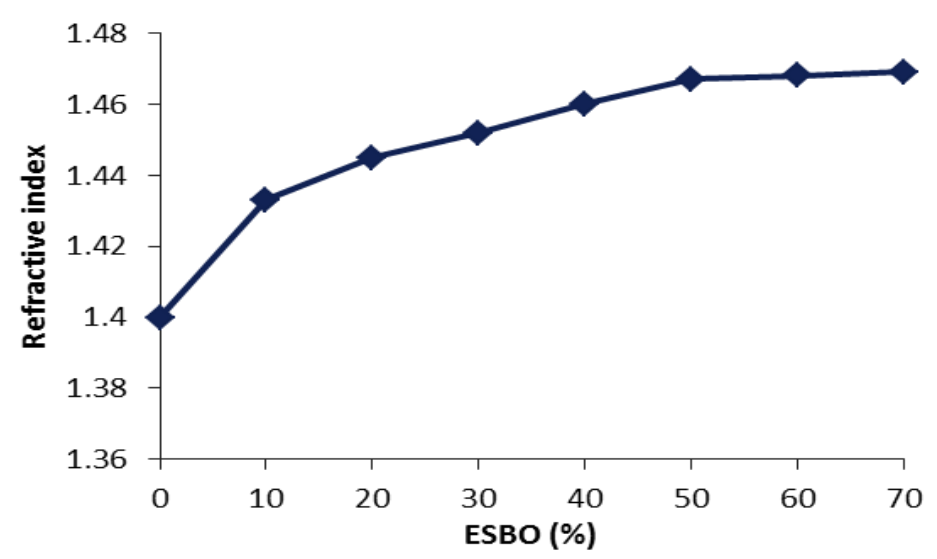

Fig. 6. Effect of ESBO content on the refractive index of ESBO/DMU blends

In Figure 7, the clear DMU resin turned colloidal as the turbidity of DMU increased on inclusion of ESBO (at 10\%). However, subsequent increase in ESBO amount in the blends did not raise the turbidity level further. This effect can be attributed to the haze imparted to the clear DMU resin on addition of ESBO which is yellowish in color. Similar effect was reported for polystyrene in DMU (Osemeahon et al. 2015).

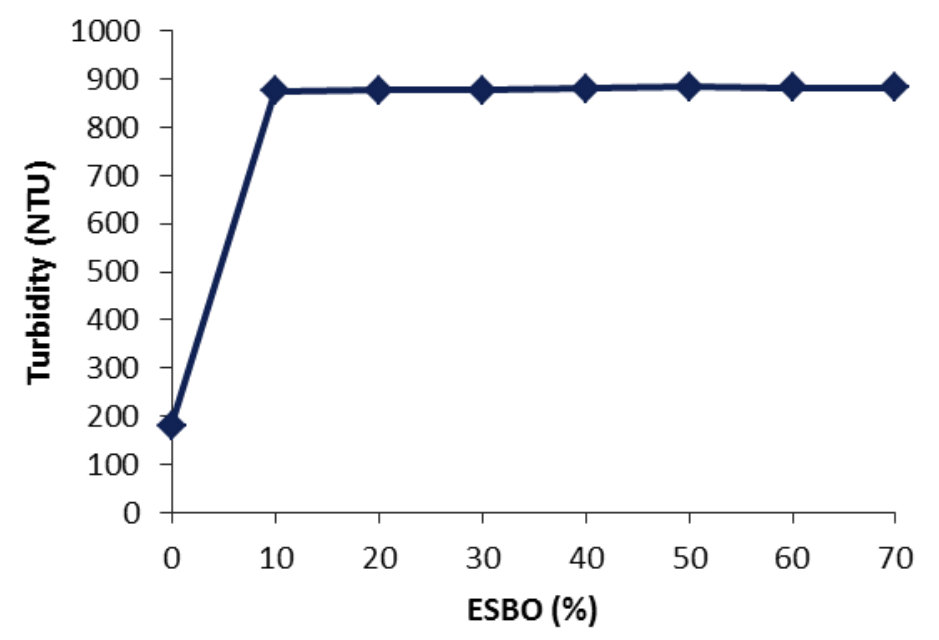

Fig. 7. Effect of ESBO content on the turbidity of ESBO/DMU blends

One of the setbacks of urea formaldehyde resin in coating application is the film's poor resistance to moisture uptake (Salthammer et al. 2010). Low moisture resistance in paint binder initiates paint failure such as blistering, 
alligatoring and brooming (Akinterinwa et al. 2015). Figure 8 shows that there was a sharp decrease in the amount of moisture uptake when ESBO was included in DMU (at 10\%), followed by a gradual decrease as the amount of ESBO increases in the blends. The sharp decrease in moisture uptake can be attributed to the formation or substitution with hydrophobic functionalities form the interaction between ESBO and DMU (Cakir et al. 2012). This interaction can be said to quickly approach equilibrium with increase in the ESBO amount in the blends.

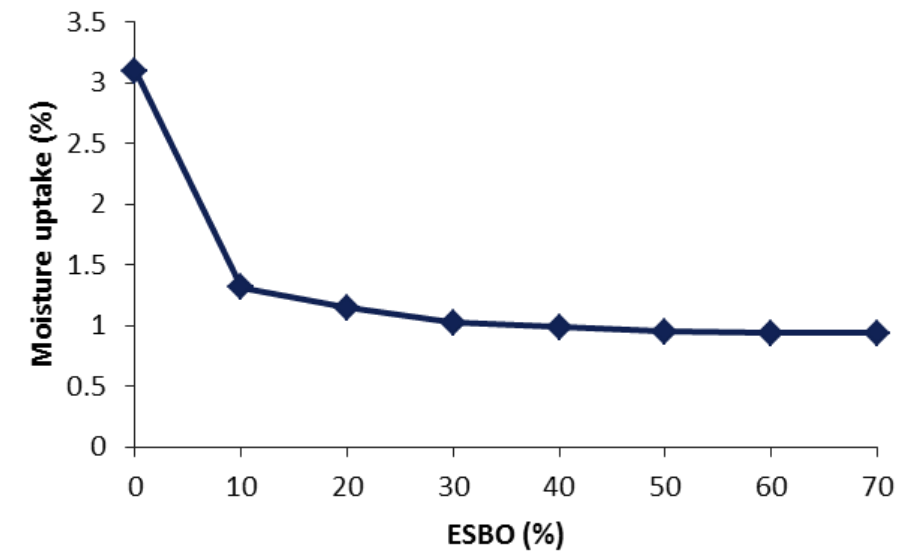

Fig. 8. Effect of ESBO content on the moisture uptake of ESBO/DMU blends

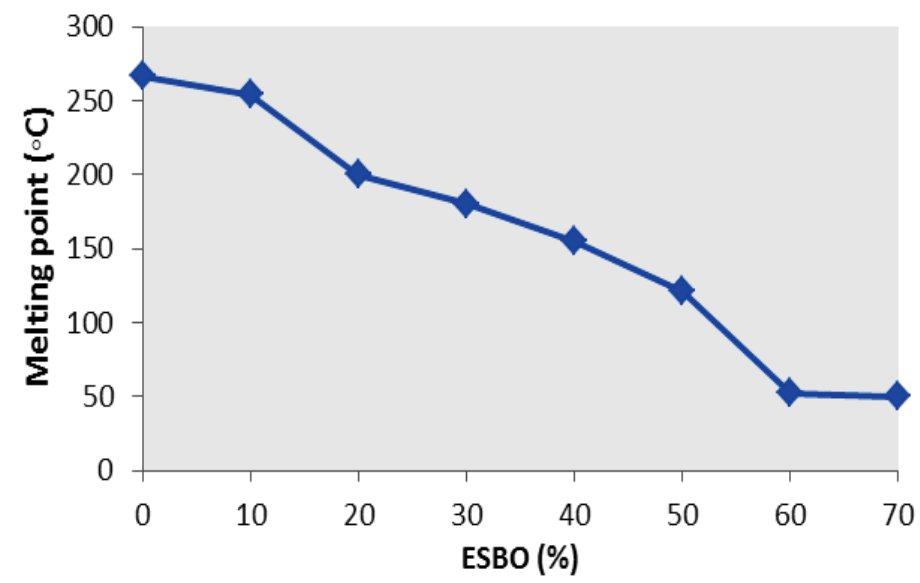

Fig. 9. Effect of ESBO content on the melting point of ESBO/DMU films

Dry film of urea formaldehyde is hard and prone to brittleness, therefore, impacting softness/flexibility is a favorable modification of the resin. Melting point of polymers is related to flexibility (Yildiz et al. 2007). Figure 9 shows 
the effect of ESBO concentration on the melting temperature of ESBO/DMU blends' films. The melting temperature of the blends' films decreased with increase in the amount of ESBO till 50\%, after which it drops sharply. This shows that the blends' films increased in flexibility as the ESBO content increases. Above 50\%, ESBO's soft film forms bulk of the heterogeneous films, this attributes to the low melting temperatures.

Formaldehyde emission during resin cure is another drawback in urea formaldehyde's application in coating (Salthammer et al. 2010, Akinterinwa et al. 2015). Figure 10 shows the effect of ESBO on formaldehyde emission from blends. Formaldehyde emission from the blends decreases as the ESBO content was increased till 50\%, after which the emission remains relatively constant. Reduction in formaldehyde emission may be expected due to the reduction in the amount of DMU present in the blends as the ESBO content increases. However, the rapid reduction in emission on inclusion of ESBO can be attributed to interactions involving the hydroxyl end of DMU, hindering condensation reactions leading to the emission of formaldehyde (Osemeahon et al. 2015). Optimum formaldehyde emission (at $40 \% \mathrm{ESBO}$ ) is $0.045 \mathrm{ppm}$, this is appreciably lower than the $0.08 \mathrm{ppm}$ maximum acceptable level in the coating industry (Salthammer et al. 2010).

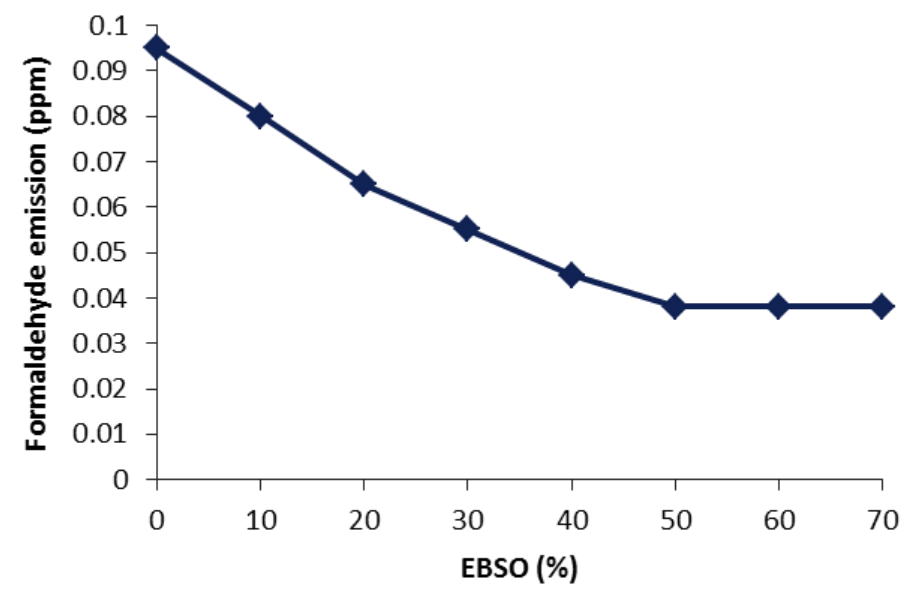

Fig 10. Effect of ESBO content on formaldehyde emission of ESBO/DMU films.

Gel or gelation time is the time taken by a substance in a fluid state to dry or set into a rigid structure with mechanical properties. Figure 11 shows that the gel time decreases with increase in ESBO content in the blend till 50\%, after which the dry time increased again. 


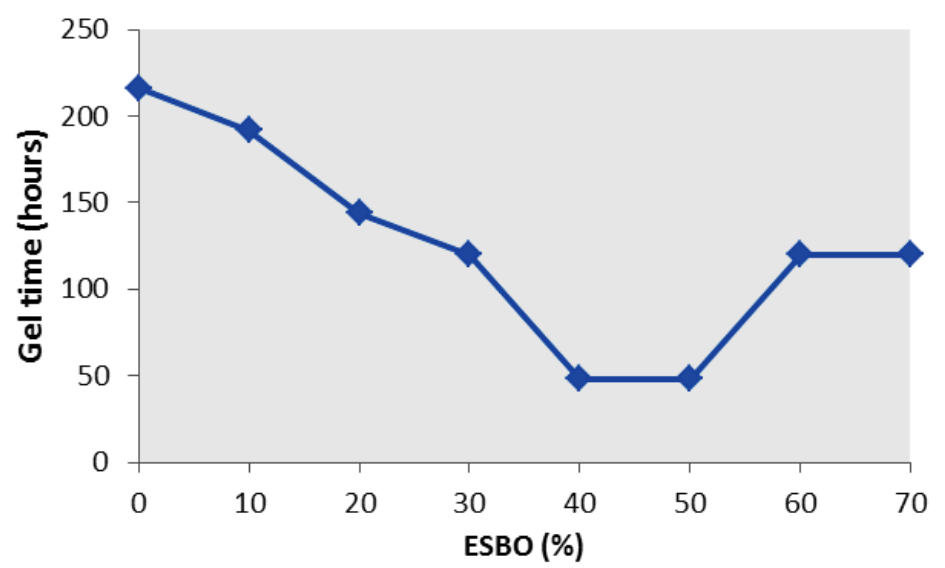

Fig 11. Effect of ESBO content on the gelation time of ESBO/DMU films

This effect can be attributed to an efficient blending/interaction between ESBO and DMU resulting in the formation of a substance with unique mechanical properties compare to ESBO and DMU.

To check the tenability of the blends as possible binder for emulsion paint, its solubility or stable disposability in water was recorded in Table 1 . ESBO/DMU blends uniformly disperse in water up to 40\% ESBO, above which there was decline in stable disposability in water. This is due to the oil phase which increasingly forms the bulk phase of the blends with ESBO more than $40 \%$.

Table 1: Effect of ESBO content on the solubility of ESBO/DMU blends.

\begin{tabular}{cl}
\hline Concentration (\%) & Solubility \\
\hline 0 & Soluble \\
10 & Soluble \\
20 & Soluble \\
30 & Soluble \\
40 & Soluble \\
50 & Sparingly soluble \\
60 & Insoluble \\
70 & Insoluble \\
\hline
\end{tabular}


Table 2. Comparison of some properties of ESBO/DMU (40\%) with other paint binders.

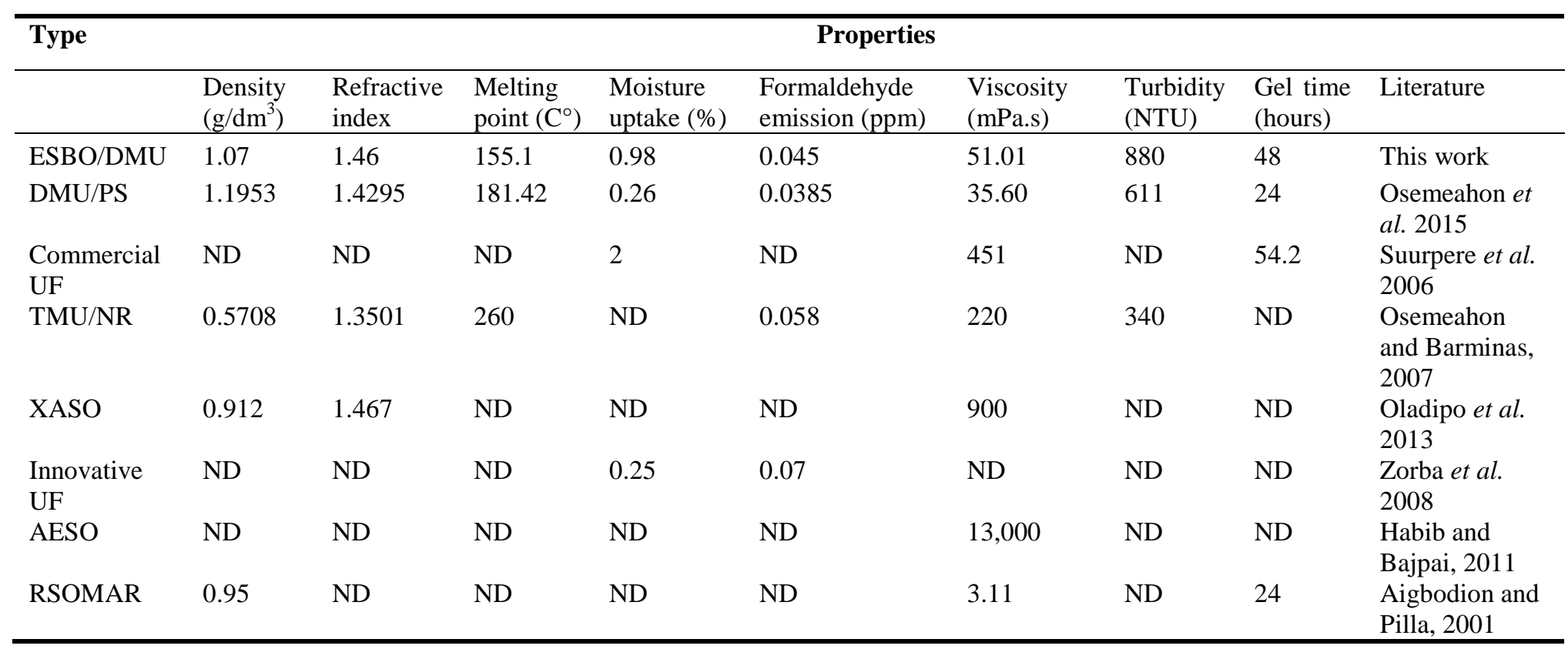

Key: TMU: Trimethylol Urea, PS: Polystyrene, UF: Urea Formaldehyde, NR: Natural Rubber, XASO: Ximenia americana Seed Oil, AESO: Acrylated Epoxidized Soybean Oil, ER: Epoxy Resin, RSOMAR: Rubber Seed Oil Modified Alkyd Resin, ND: Not Determined. 


\subsection{Comparison of ESBO/DMU binder with other reported binders}

Being of optimum properties, Table 2 compares 40\% ESBO/DMU blend with those of some reported binders. Looking through the data presented on Table 2, it can be said that ESBO/DMU at optimum blend has good potentials to compete among other emulsion paint binders recommendable to the coating industry.

\section{Conclusions}

Soybean oil was epoxidized to obtain ESBO with 2.56\% oxirane oxygen, 67\% iodine value conversion and a distinct oxirane oxygen IR peak. ESBO blended homogeneously with DMU resin. Blends with 10 to 50\% ESBO in DMU forms a stable continuous phase, and a reaction between ESBO and DMU (40 $\% \mathrm{ESBO} / \mathrm{DMU}$ ) was suspected for the disappearance of the oxirane oxygen peak in the FTIR spectra of the blend. Increase in the amount of ESBO in ESBO/DMU blends, decrease formaldehyde emission to safe levels, decrease moisture absorption by the blend film, impact softness/ flexibility as interpreted from the reduction in the melting point of the blend film, but decrease homogeneous disposability/solubility in water. The blend at $40 \%$ ESBO/DMU was selected for optimum properties. A comparison of the product with other reported binders shows that it has good potentials to compete in the coating industry as an efficient emulsion paint binder.

\section{Acknowledgements}

Authors appreciate Mr. Isaku Maizana and the entire laboratory technologists of the Department of Chemistry Modibbo Adama University of Technology, Yola, Nigeria. Comments from anonymous reviewers are acknowledged.

\section{References}

Aigbodion AI, Pillai CKS. 2001. Synthesis and molecular weight characterization of rubber seed oil-modified alkyd resins. Journal of Applied Polymer Science 79(13): 2431-2438.

Akinterinwa A, Osemeahon SA, Nkafamiya II, Dass PM. 2015. Formulation of Emulsion Paint from a Copolymer Composite of Dimethylol Urea/Polystyrene. Chemistry and Materials Research 7(7): 20-26.

AOAC. 2000. Official method of analysis of AOAC international. Eds: $17^{\text {th }}$ Association of official Analytical chemists (AOAC), Washington DC.

Barminas JT, Osemeahon SA. 2007. Development of Amino Acid Resin for Paint Formulation: Copolymerization of Methylol Urea with Polyesther. African Journal of Biotechnolgy 6(12): 1432-1440. 
Cakir M, Kartal I, Demirer H, Samur R. (2012). Effect of water absorption on the wear behavior of sol-gel processed epoxy/silica hybrids. Scientific Research and Essays 7(7): 805812.

Cheng W, Liu G, Wang X, Liu X, Jing L. 2015. Kinetics of the epoxidation of soybean oil with $\mathrm{H}_{2} \mathrm{O}_{2}$ catalyzed by phosphotungsticheteropoly acid in the presence of polyethylene glycol. European Journal of Lipid Science and Technology 117(8): 1185-1191.

Edoga MO. 2006. Ethylated Urea-Ether-Modified Urea-Formaldehyde Resins, Part I: Structural and Physicochemical Properties. Leonardo Electronic Journal of Practices and Technologies 9: $121-136$.

Fink JK. 2013. Reactive Polymers Fundamentals and Applications: A Concise Guide to Industrial Polymers. William Andrew.187 pp.

Habib F, Bajpai M. 2011. Synthesis and characterization of acrylated epoxidized soybean oil for UV cured coatings. Chemistry and Chemical Technology 5(3): 317-326.

Idowu-Oyewole ET, Osemeahon, SA, Dass PM, Akinterinwa A. 2016. Synthesis and Characterization of an Emulsion Paint Binder from a Copolymer Composite of Trimethylol Urea/Polyethylene. International Journal of Materials Science and Engineering 4(2): 161171.

Liem H, Etchegoin P, Whitehead KS, Bradley DDC. 2002. Raman scattering as a probe of morphology in conjugated polymer thin films. Journal of Applied Physics 92(2): 1154-1161.

Oladipo GO, Eromosele IC, Folarin OM. 2013. Formation and characterization of paint based on alkyd resin derivative of Ximeniaamericana (wild olive) seed oil. Environment and Natural Resources Research 3(3): 52.

Osemeahon SA, Barminas JT. 2007. Study of a composite from reactive blending of methylol urea resin with natural rubber. African Journal of Biotechnology 6(6): 810-817.

Osemeahon SA, Nkafamiya II,Maitera ON, AkintreinwaA. 2015. Synthesis and characterization of emulsion paint binder from a copolymer composite of dimethylol urea/polystyrene. Journal of Polymer and Composites 3(2): 11-21.

Pantone V, Annese C, Fusco C, Fini P, Nacci A, Russo A, D’Accolti L. 2017. One-Pot Conversion of Epoxidized Soybean Oil (ESO) into Soy-Based Polyurethanes by $\mathrm{MoCl}_{2} \mathrm{O}_{2}$ Catalysis. Molecules 22(2): 333.

Pizzi A, Beaujean M, Zhao C, Properzi M, Huang Z. 2002. Acetal-induced strength increases and lower resin content of MUF and other polycondensation adhesives. Journal of Applied Polymer Science 84(13): 2561-2571.

Saithai P, Lecomte J, Dubreucq E, Tanrattanakul V. 2013. Effects of different epoxidation methods of soybean oil on the characteristics of acrylated epoxidized soybean oil-co-poly (methyl methacrylate) copolymer. Express Polymer Letters 7(11): 910-924.

Salthammer T, Mentese S, Marutzky R. 2010. Formaldehyde in the indoor environment. Chemical Reviews 110(4): 2536-2572.

Siggia S. 1963. Quantitative organic analysis, eds: 3rd John Wiley \& Sons, New York.103 pp.

Suurpere A, Christjanson P, Siimer K. 2006. Rotational viscometry for the study of ureaformaldehyde resins. Proceedings of the Estonian Academy of Sci Engineering 12(2): 134146.

Vilas JL, Laza JM, Garay MT, Rodriguez M, Leon LM. 2001. Unsaturated polyester resins cure: Kinetic, rheologic, and mechanical-dynamical analysis. I. Cure kinetics by DSC and TSR. Journal of Applied Polymer Science 79(3): 447-457.

Zorba T, Papadopoulou E, Hatjiissaak A, Paraskevopoulos K, Chrissafis K. 2008. Ureaformaldehyde resins characterized by thermal analysis and FTIR method. Journal of Thermal Analysis and Calorimetry 92(1): 29-33. 